\title{
CHLORIDES IN THE CEREBRO-SPINAL FLUID IN CASES OF MENINGITIS
}

BY

\author{
F. S. FOWWEATHER, M.D., M.Sc., F.I.C., D.P.H.
}

(From the Department of Pathology and Bacteriology, University of Leeds).

Since the discovery by Mestrezat that the chlorides of the cerebro-spinal fluid are reduced in meningitis, and to the greatest extent in tuberculous meningitis, a considerable amount of work has been devoted to this subject. As a result of the experience gained from this work the original claim of Mestrezat that values for chloride exceeding 0.64 per cent. absolutely exclude the diagnosis of tuberculous meningitis is no longer justified; the sharp distinction at first believed to be present between the results obtained in tuberculous meningitis and those found in the meningitis of other types does not exist.

I have examined the results obtained in this laboratory during the last three years, in cases of children, as well as the results published by other workers, and have arrived at the following conclusions :-

1. Reduction of chlorides is almost a constant finding in meningitis.

2. This reduction occurs, in general, to a greater degree in tuberculous meningitis than in other forms of meningitis.

So far as the analytical results themselves are concerned it may be said that :-

(a) Values below 0.59 per cent. are frequently found in tuberculous meningitis, but only very rarely in other forms. Linder and Carmichael $^{1}$ quote a case of chronic meningococcal meningitis in which the chloride was 0.52 per cent.

(b) Values between 0.59 per cent. and 0.64 per cent. are much more frequently found in tuberculous meningitis than in other forms.

(c) Values between 0.64 per cent. and 0.69 per cent., generally characteristic of acute purulent meningitis, may be found fairly frequently in tuberculous meningitis especially in the beginning of the illness, in which, however, there is often a rapid fall in chloride value with the progress of the disease.

Among the cases of meningitis which I have investigated, two appear to merit special attention, since they show that the reduction of the chloride may. be the first change to be found in the cerebro-spinal fluid in such cases. This is a finding to which $I$ have not previously seen any reference, but it appears to have some value from the point of view of diagnosis.

The most constant change in the cerebro-spinal fluid in meningitis has been considered to be an increase in the number of cells, and in the absence of such 
an increase a diagnosis of meningitis would very rarely be made. No doubt such an increase can be demonstrated in a very great majority of cases and probably would occur in all cases at some stage of the disease if the patient lived long enough. But the two cases recorded here show that the appearance of this change is not absolutely constant ; it may in some cases be delayed, so that the reduction of chloride precedes it. The details of the cases are as follows :-

Case 1. Boy, aged 1 year, 8 months. An examination of the cerebro-spinal fluid on the day after admission showed 3 lymphocytes per c.mm. A further examination next day showed 4 lymphocytes per c.mm. On this occasion a chloride determination was carried out, the result being 0.62 per cent. Death occurred on the third day after admission.

A post-mortem examination by Prof. M. J. Stewart showed primary tracheo-bronchial gland tuberculosis; advanced tuberculous basal meningitis with internal hydrocephalus; acute miliary tuberculosis of lungs, liver and kidneys.

Case 2. Boy, aged 1 year. Two days after admission the cerebro-spinal fluid was found to contain 3 lymphocytes per c.mm. Eight days after admission 4 lymphocytes per c.mm. were found, while the chloride was found to be 0.65 per cent. Three days afterwards, in a fluid obtained shortly before death, about 2,500 cells per c.mm., mainly polymorphonuclear leucocytes, were found, while the chloride amounted to $0 \cdot 62$ per cent.

A post-mortem examination by Dr. R. H. Morley showed broncho-pneumonia; right temporo-sphenoidal abscess; generalized meningitis.

In the first case the reduction of chlorides, was the only definite evidence of meningitis found in the cerebro-spinal fluid; while in the second, reduction of chlorides, indicated the spread of inflammation from an abscess, causing generalized meningitis, before any indication of this condition was given by the cell count.

A third case in which no chloride estimations were made indicates the variability of the cell count in meningitis, and shows that normal counts may be obtained when meningitis is undoubtedly present.

Case 3. Girl, aged $3 \frac{1}{2}$ years. On the day after admission the cerebro-spinal fluid contained 15 lymphocytes per c.mm. Six days later, only 4 lymphocytes per c.mm. were found. Death occurred on the day after the latter examination.

A post-mortem examination by Prof. Stewart showed tuberculous meningitis; fairly advanced caseous tuberculosis of the mesenteric glands; a few miliary tubercles in the lungs.

In this case, while the first examination certainly showed an increase in the cell count, the result of the second examination was approximately normal, and if only the latter examination had been made no evidence of meningitis would have been obtained from the cerebro-spinal fluid. If a chloride determination had been made it is probable that a reduced value would have been found.

Nowicka ${ }^{2}$ quotes a case in which a fluid was found to be normal so far as cells, albumin and sugar were concerned, but only 0.62 per cent. of chlorides, was present. The patient was a girl of 10 years, who had had Pott's disease some time previously. She was seriously ill when admitted, showing wasting, marked dyspnœa and generalized cyanosis. The temperature showed large, irregular variations, and there were abundant signs of disease in the lungs. She was considered to be suffering from acute tuberculosis. Death occurred after two weeks illness. No mention is made of any post-mortem examination.

Nowicka considers that the low chlorides in this case simply indicate a general reduction of chlorides throughout the body, but in view of the cases 
mentioned above it seems probable that it may have been an early indication of the onset of tuberculous meningitis, for such a development as a terminal stage in tuberculous disease in children is, of course, of fairly frequent occurrence.

Linder and Carmichael ${ }^{1}$ in a study of four cases of meningitis obtained results tending to show that the reduction of the chlorides in the cerebro-spinal fluid is accounted for by a parallel drop in the blood chlorides. It wouldtherefore follow that low chlorides should be found in the cerebro-spinal fluid in any case in which the serum chlorides are reduced. But the frequency with which this occurs in conditions other than meningitis is not known, except in lobar pneumonia, in which low blood and cerebro-spinal fluid chlorides are the rule, and is probably not great ; whereas in meningitis the occurrence of low chlorides in the cerebro-spinal fluid is almost constant. As Greenfield ${ }^{3}$ pointed out recently, the present views of the actual mechanism of the reduction of chloride in the cerebro-spinal fluid do not alter the significance of such a reduction from the prognostic standpoint : nor, I believe, do they make much difference to its significance with regard to diagnosis.

\section{Conclusions.}

The conclusions to be drawn from the cases I have described are :-

1. A normal cell-count in the cerebro-spinal fluid is not incompatible with the presence of meningitis.

2. A reduction of the chlorides in the cerebro-spinal fluid may be the first alteration to be found in cases of meningitis. In suspected cases, therefore, where the cell count is found to be normal, a chloride determination should be made before the diagnosis of meningitis is excluded.

I wish to express my thanks to Dr. C. W. Vining for his permission to use the records of cases under his care.

\section{REFERENCE.}

1. Linder, G. C., \& Carmichael, E. A., Biochem. J., Camb., 1928, XXII, 46.

2. Nowicka, H., Arch. de méd. d. enf., Paris, 1924, XXVII, 726.

3. Greenfield, J. G., Brit. Med. J., Lond., 1929, ii, 84]. 